\title{
Review
}

\section{Synaptic Failure: Focus in an Integrative View of ALS}

\author{
Caty $\operatorname{Casas}^{\mathrm{a}, *}$, Raquel Manzano ${ }^{\mathrm{b}, 1}$, Rita Vaz $^{\mathrm{c}, 1}$, Rosario Osta $^{\mathrm{b}}$ and Dora Brites ${ }^{\mathrm{c}}$ \\ ${ }^{a}$ Group of Neuroplasticity and Regeneration, Institut de Neurociències and Department of Cell Biology, \\ Physiology and Immunology, Universitat Autònoma de Barcelona, and Centro de Investigación Biomédica \\ en Red sobre Enfermedades Neurodegenerativas (CIBERNED), Bellaterra, Barcelona, Spain \\ ${ }^{\mathrm{b}}$ Laboratory of Genetic Biochemistry (LAGENBIO-I3A), Aragón Institute of Health Sciences, Universidad de \\ Zaragoza, Zaragoza, Spain \\ ${ }^{\mathrm{c}}$ Research Institute for Medicines (iMed.ULisboa), Faculdade de Farmácia, Universidade de Lisboa Lisbon, \\ Portugal; Department of Biochemistry and Human Biology, Faculdade de Farmácia, Universidade de Lisboa \\ Lisbon, Portugal
}

\begin{abstract}
From early description by Charcot, the classification of the Amyotrophic Lateral Sclerosis (ALS) is evolving from a subtype of Motor Neuron (MN) Disease to be considered rather a multi-systemic, non-cell autonomous and complex neurodegenerative disease. In the last decade, the huge amount of knowledge acquired has shed new insights on the pathological mechanisms underlying ALS from different perspectives. However, a whole vision on the multiple dysfunctional pathways is needed with the inclusion of information often excluded in other published revisions. We propose an integrative view of ALS pathology, although centered on the synaptic failure as a converging and crucial player to the etiology of the disease. Homeostasis of input and output synaptic activity of MNs has been proved to be severely and early disrupted and to definitively contribute to microcircuitry alterations at the spinal cord. Several cells play roles in synaptic communication across the MNs network system such as interneurons, astrocytes, microglia, Schwann and skeletal muscle cells. Microglia are described as highly dynamic surveying cells of the nervous system but also as determinant contributors to the synaptic plasticity linked to neuronal activity. Several signaling axis such as TNF $\alpha$ /TNFR1 and CX3CR1/CX3CL1 that characterize MN-microglia cross talk contribute to synaptic scaling and maintenance, have been found altered in ALS. The presence of dystrophic and atypical microglia in late stages of ALS, with a decline in their dynamic motility and phagocytic ability, together with less synaptic and neuronal contacts disrupts the MN-microglia dialogue, decreases homeostatic regulation of neuronal activity, perturbs "on/off" signals and accelerates disease progression associated to impaired synaptic function and regeneration. Other hotspot in the ALS affected network system is the unstable neuromuscular junction (NMJ) leading to distal axonal degeneration. Reduced neuromuscular spontaneous synaptic activity in ALS mice models was also suggested to account for the selective vulnerability of MNs and decreased regenerative capability. Synaptic destabilization may as well derive from increased release of molecules by muscle cells (e.g. NogoA) and by terminal Schwann cells (e.g. semaphorin $3 \mathrm{~A})$ conceivably causing nerve terminal retraction and denervation, as well as inhibition of re-connection to muscle fibers. Indeed, we have overviewed the alterations on the metabolic pathways and self-regenerative capacity presented in skeletal muscle cells that contribute to muscle wasting in ALS. Finally, a detailed footpath of pathologic changes on MNs and associated dysfunctional and synaptic alterations is provided. The oriented motivation in future ALS studies as outlined in the present article will help in fruitful novel achievements on the mechanisms involved and in developing more target-driven therapies that will bring new hope in halting or delaying disease progression in ALS patients.
\end{abstract}

Keywords: ALS, muscle, microglia, motor neuron, synapses, neuromuscular junction

${ }^{*}$ Correspondence to: Caty Casas Louzao, Unitat de Fisiologia Mèdica, Edif. M, Universitat Autònoma de Barcelona, E-08193 Bellaterra, Barcelona, Spain. Tel.: +34 935811324; Fax: +34 935812986; E-mail: Caty.Casas@uab.cat.

${ }^{1}$ Both authors contribute equally. 


\section{HISTORY, PATHOLOGICAL FEATURES AND ALS-ASSOCIATED GENETIC MUTATIONS}

Jean-Martin Charcot in 1869 [1] was the first physician to clearly isolate amyotrophic lateral sclerosis (ALS) from the other sclerosis by linking symptoms to motor neurons (MNs) that originate in the spinal cord (SC) $[2,3]$. At the moment, even if we have no marker for diagnosis or prognosis, the disease is recognized by the severe degeneration of lower (spinal and bulbar) and upper (corticospinal) MNs [4] although clinical presentations may differ. It is considered a rare disease with an incidence from 1.2 to 4 per 100,000 patients in Caucasians [5, 6]. Approximately two thirds of ALS patients have a spinal form of the disease (limb onset) and evidence symptoms related to focal muscle weakness and wasting which may start either distally or proximally in the upper and lower limbs. Gradually, spasticity may develop in the weakened atrophic limbs, affecting manual dexterity and gait. ALS patients with bulbar onset usually complain from dysarthria and dysphagia for solids or liquids, with limb symptoms developing either simultaneously with bulbar symptoms or within 1-2 years. Paralysis is progressive and leads to death due to respiratory failure within 2-3 years for bulbar onset cases and within 3-5 years for limb onset [7]. Although initially characterized by this progressive muscle atrophy, the term "lateral sclerosis" refers to a distinctive anatomo-pathological trait of hardening of the anterior and lateral corticospinal tracts, the major efferent motor conduits to the spinal cord, and alterations in the corpus callosum, [8] suggesting that it is a complex multi-component disease.

This simple vision of considering ALS as a type of $\mathrm{MN}$ disease has been challenged nowadays due to the evidences resulting from different sources of research. Applications of neuroimaging such us magnetic resonance imaging (MRI), magnetic resonance spectroscopy or diffusion tensor imaging have revealed the involvement of diffuse brain presentations. At cortical level, premotor and motor regions seem dominant although frontal and anterior temporal regions, in addition to subcortical pathways, are also key structures [9]. Because motor planning, motor abstraction, emotional expression, and language are all affected in ALS, some authors demand that ALS should be considered as a result of a system degeneration due to network failure instead of focusing on individual neuronal populations [10].
From the systemic point of view, ALS seems to be restricted not only to the nervous system but also to include whole-body physiology. In particular, energy homeostasis is compromised in patients with ALS, which has notable clinical implications such as weight loss, hypermetabolism, and hyperlipidaemia. These abnormalities correlate with the duration of survival [11]. Finally, more recently, some authors highlight alterations in all the compounds of the neurovascular unit [12], comprising the existence of interactions between the vascular and neural cells [13] in ALS patients and animal models [14-16] as contributors to the disease pathogenesis. Despite MN cell loss is the critical hallmark during the course of the disease [17], other cells are considered major determinants in the onset and ALS progression such as astrocytes [18], microglia [19], oligodendrocytes [20], muscle cells [21], or accepted as contributors, such as lymphocytes [22, 23], pericytes [24] and interneurons [25].

Besides these changes in the physiopathological perspective of ALS, another important source of new evidences comes from genetic research. Mutations in the gene encoding the ubiquitously expressed $\mathrm{Cu} / \mathrm{Zn}$ superoxide dismutase (SOD1), the first discovered in 1993 [26], were found to cause the familial ALS (fALS) in approximately 10 to $20 \%$ of ALS cases [27]. Since then, 164 mutations have been found in such gene [28]. It is at present unclear whether all mutations are pathogenic but studies producing at their understanding have produced a large amount of data thanks to the generation of ALS transgenic models carrying those mutations [29]. The transgenic SOD1 mouse carrying the substitution of glycine at residue 93 by alanine $\left(\operatorname{tgSOD} 1^{\mathrm{G} 93 \mathrm{~A}}\right)$ is the most widely studied model of ALS [30]. These mice are normal at birth, but begin to develop $\mathrm{MN}$ dysfunctions at 11 weeks of age that progress from hindlimbs to forelimbs presenting as weakness, tremors, reduced extension reflex, total paralysis, and, finally, death $[31,32]$. Several transgenic mouse strains expressing the human SOD1 gene with different mutations, have been generated to date for studying the disease pathogenesis [33] that also present embryonic and neonatal abnormalities in MNs and SC circuitry far earlier than any symptomatic manifestation appear [34,35]. Later on, other genes were reported to be implicated as well, which have been recently reviewed [36]. To date, 47 missense and one truncating mutations were found in fALS and sporadic (sALS) patients [27]. Among these genes, mutations in TAR-DNA binding protein 
(TARDBP or TDP-43) and fused in sarcoma (FUS) [27] have revealed its importance in the degeneration of MNs and in the so called continuum between ALS and Fronto-Temporal Lobar Degenerationa (FTLD), which is present in $45 \%$ of cases of ALS [37]. Recently, it was suggested that TDP-43 dysfunction is mainly localized in SC astrocytes, which will trigger $\mathrm{MN}$ degeneration [38]. Indeed, astrocytes expressing mutant SOD1 and TDP-43 were shown to cause the death of MNs trough a common pathogenic pathway that involves nitrosative stress [39]. Intriguingly, measurements of TDP-43 in cerebrospinal fluid (CSF) and blood revealed its limited role as a diagnostic tool [40]. Finally, the most common genetic abnormality in in families with FTLD or ALS has recently been mapped to a non-coding repeat expansion in the gene C9ORF72 at 9p21 (reviewed in [41]) Strikingly, this hexanucleotide repeat accounts for up to $40 \%$ of fALS and $7 \%$ of sALS [5]. The contribution of all these mutations to the pathology is mentioned in the section below.

Thus, ALS turns out to be a multi-systemic, non-cell autonomous and complex neurodegenerative disease that requires an integrative vision. In the last decade the huge amount of knowledge acquired has shed insights into the pathological mechanisms underlying ALS from different perspectives such as genetics and proteomics, and longitudinal studies have contributed to improve our understanding on disease progression. However, more than ever, is time to have a whole vision on the several determinants implicated in ALS to better focus future research on identifying new targets and discovering novel efficacious therapies that make a substantial difference for the outcome of ALS patients. Although pretending to draw an integrative vision to ALS pathology and complexity we always kept in mind the synaptic failure as an important contributor to the etiology of the disease.

\section{ETIOPATHOGENESIS: DYING BACK, DYING FORWARD AND SYNAPTIC FAILURE HYPOTHESIS}

Determining the nature of the etiopathogenesis in ALS is a difficult task. This is an issue under intense debate nowadays due to the importance of targeting a specific tissue for therapy. The debate has been focused in the direction of the spreading of the disease in the central nervous system (CNS) since both cortical and spinal regions are affected. The so named "Dying-Back" and "Dying-Forward" hypothesis co-exist to explain the pathogenic pathways for degenerative spreading. Both are based on a network perspective that explains a decline of structural integrity with preserved functional organization of the motor network in ALS. While "Dying-Forward" hypothesis claim that cortical hyperexcitability in cortical motor areas might trigger anterograde degenerative process towards the SC, the "Dying-Back" theory of pathological spread in ALS suggests that early degeneration is more likely to be captured at the spinal anterior horn due to weakness in neuromuscular connectivity rather than in the brain [42]. Cortical regions mostly affected include the precentral gyrus, especially in subregions with maximal clustering of cortical MNs (Betz cells), whose degeneration is considered to be a pathological hallmark of ALS [43]. Regions functionally connected with the motor cortex are deeply affected with a reduced microstructural organization of the efferent conduits that form the corticospinal tracts, interconnecting the motor network. This functional connectivity may allow the rapid spread of the disease. Indeed, it has been recently hypothesized that disease might spread contiguously or non-contiguously following a 'domino-prion-like' propagation between neighboring neurons in a cell autonomous or non-cell autonomous manner. This supposition is inspired in the sticky properties of mutant SOD1, and normal TDP43, which can undergo a seeded aggregation similar to prions. Both native and mutant forms of SOD1 associated with each other easily form aggregates and fibrils under denaturing conditions [44]. Importantly, removal of the misfolded seeds does not stop aggregation of endogenous SOD1, indicating that the newly formed aggregates can act as templates for the subsequent misfolding of additional native SOD1 [45] (reviewed in [46]). However, Dying-Forward hypothesis might not be easy to demonstrate in mouse models due to significant differences in connectivity between mice and human. Betz cells in humans connect monosynaptically to spinal MNs through ventral corticospinal track. However, this association is very scarce in mouse whose corticospinal track course mainly through the dorsal funiculi and end on interneurons at lamina $\mathrm{V}$ of the dorsal horn at the SC. Only few axons run also through dorsolateral and ventral funiculi which may contact directly to some MNs [47]. Although several alterations have early been observed in cortical MNs in parallel to spinal MNs, it might be improbable that this will 


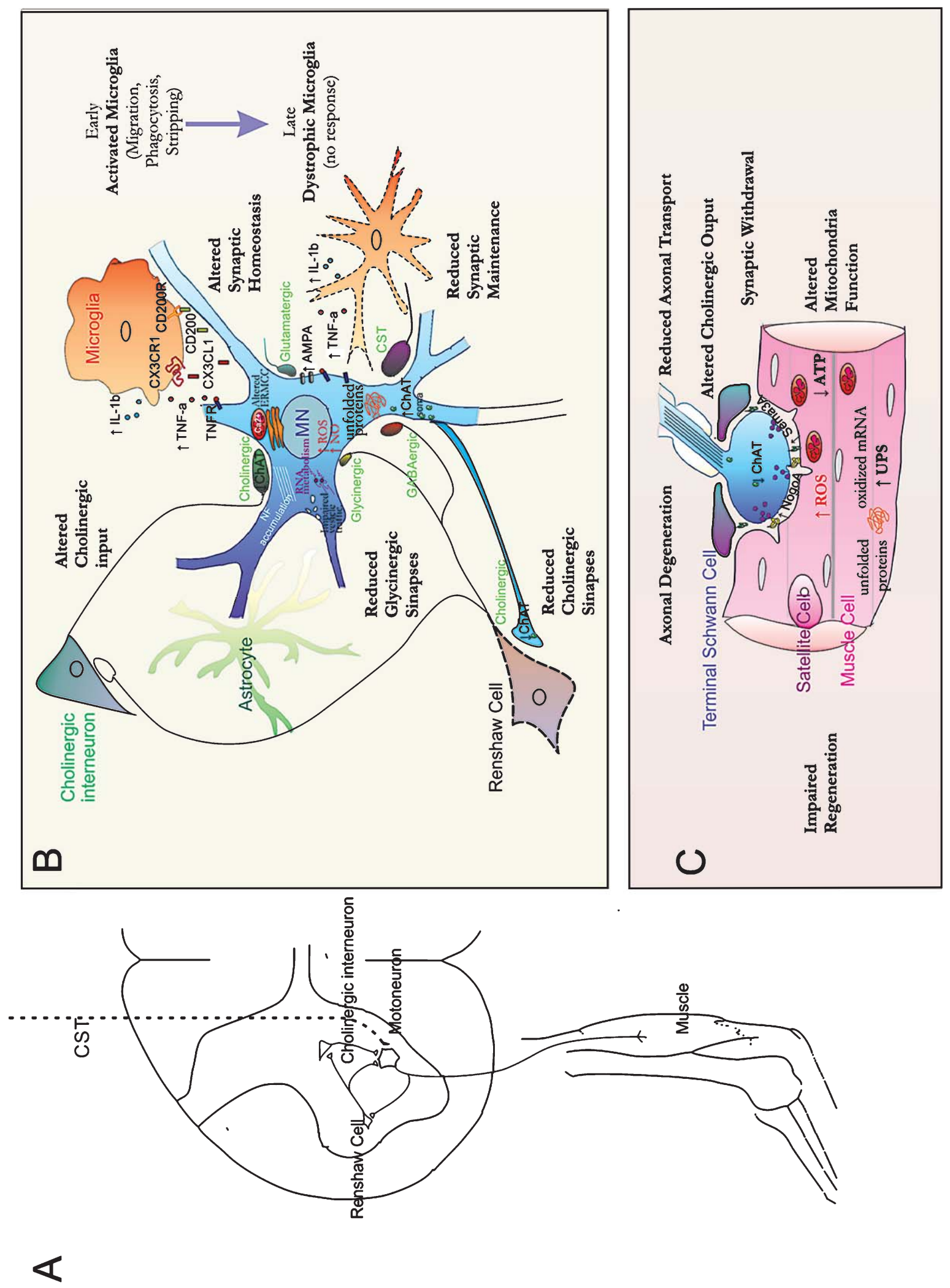


occur as one being the consequence of the other in mouse models of ALS. It would be more plausible that noxa induction, with a crucial role in apoptosis and MN degeneration [48, 49] will occur specifically and importantly mainly in MNs, independently of their location. This favors the hypothesis of a complex genetic-environmental interaction as the causal factor for MN degeneration. Factors may not only affect MNs, but also other cells in a less degree, which together certainly account for a toxic neuronal and system microenvironment. Among these factors, several have been reported to be likely contributors to the disease such as the exposure to tobacco or some pesticides or the intense practice of athleticism or soccer as determined by epidemiological analysis [5].

Synaptic terminal degeneration is a pathological hallmark in human ALS progression. Synapse elimination occurred even in patients who had no $\mathrm{MN}$ and corticospinal tract impairment [50, 51]. The working model whereby loss of synapses at lower MNs plays an important role in ALS pathogenesis is commonly referred to as the 'synaptic stripping' hypothesis. Direct evidence in support of this hypothesis comes from studies showing a decrease in the expression of synaptic vesicle proteins like synaptophysin and synapsin and syntaxin and synaptotagmin in ALS and animal models [52, 53], which were evident in remaining $\mathrm{MNs}$ and suggest a loss of synapses before neuronal loss. Nagao and colleagues showed that expression of the synaptic marker vesicle-acetylcholine-transferase (VAChT) was reduced in the ventral horn of an ALS patient, reinforcing the loss of cholinergic synapses in lower MNs as an early event in ALS [54]. In agreement with that, it has been recently described that there is a marked reduction in choline acetyltransferase (ChAT) mRNA and protein content in the cholinergic synaptic terminals apposed onto lumbar spinal MNs at the early asymptomatic phase in the tgSOD1 ${ }^{\text {G93A }}$ ALS model [55]. Besides, this reduction was also apparent in the cholinergic synaptic terminals of the connections from MNs onto Renshaw cells. Renshaw are glycinergic and gabaergic inhibitory cells that receive input from motor axon collaterals and synapse in turn on the soma of MNs in a negative-feedback fashion (homonymous recurrent inhibition). These inhibitory terminals in MNs have been observed to be reduced, preferentially the

Fig. 1. Integrative view of ALS. A. ALS is a result of the degeneration of the motor functional circuitry mainly. In humans, the corticospinal tract (CST) allows monosynaptic connection of cholinergic Betz cells in motor cortex to spinal motor neurons (MNs). At the spinal cord, MNs forme a microcircuitry with interneurons and other MNs to accomplish the efferent motor signaling. Cholinergic interneurons at lamina $\mathrm{X}$ synapse in MNs regulate motor behavior and Renshaw inhibitory interneurons receive inputs form MN axon collaterals to synapse, that turn to MNs and conforms a negative feedback regulation. B. ALS can be considered as a multi-component disease that affects synaptic communication. Several terminal boutons that make synapses on MNs are structural or functional affected, such as glycinergic and cholinergic ones. The number of glycinergic synapses from inhibitory Renshaw cells is reduced and the density of these cells has been found decreased in later stages of ALS. Choline acetyltransferase (ChAT) content is early diminished from boutons apposed on MNs and from their terminals on Renshaw cells. Altogether these variations match with the altered recurrent inhibition found in patients and contribute to imbalance in inhibitory/excitatory homeostasis. MNs present altered molecular environment with incapability to accomplish several neuroprotective programs (such as autophagy, unfolded protein response (UPR), proteosomal activity) that lead to a failure in handling protein homeostasis and aggregation, which are related to a reduced neuronal survival. There exists a persistent dysregulation in the endoplasmic reticulum (ER)-mitochondria calcium cycle (ERMCC) that couple energy from mitochondria to protein processing in the ER and participates in AMPAmediated synaptic activity. This leads to excitotoxicity and abundant reactive oxygen species (ROS) and nitric oxide (NO) production. In addition, difficulties in vesicle trafficking may occur in soma and along the axon that difficult synaptic communication with the muscle and other cells. Thus, the machinery for local protein translation in dendrites and synapses present alterations that involves the mRNA silencing foci at which TDP-43 and FUS/TLS participate with consequent mRNA metabolism alterations. Microglia contribute to synaptic scaling and maintenance by cross-talk to MNs through TNF $\alpha$ /TNFR1, CX3CL1/CX3CR1 and CD200/CD200R axis. Activation of CX3CR1 in microglia are part of the "off" signals that switch microglia from the activated state to the resting one, which may be interrupted in ALS, at least in some of the ALS stages. Indeed, in later stages, microglia become non-responsive and dystrophic, a phenotype that follows the chronic activation and the release of pro-inflammatory cytokines, such as IL1-beta and TNF-alpha. Activation of TNFR1 in neurons classically lead to apoptosis but also increases AMPA receptors at the membrane. Besides, the composition and editing of AMPA receptors which is altered in ALS contribute to excitotoxicity due to changes in their ion permeability. C. Neuromuscular junction (NMJ) fragility is promoted by disturbances in several cellular components and lead to impaired MN transmission and regeneration. At the axonal terminal, the transport is altered and, in particular, there is a reduced ChAT transport which can lead to a decrease in immediately available transmitter pool, as well as to a reduced spontaneous acetylcholine release that weakens NMJ. Synaptic withdrawn may be induced by the release of inhibitors of regeneration like Nogo A or Semaphorin 3A from muscle or terminal Schwann cells, respectively. Myofiber atrophy is one of the earliest events in ALS. Muscle cell metabolism and regeneration is altered. There exists mitochondria function defects promoting ATP reduction, disturbances of calcium buffering capacity and oxidative stress exrcerbated by muscle training and muscle hypermetabolism that leads to a rise in oxygen species, which contribute to oxidation of mRNAs and altered miRNA biogenesis. These features contribute to a reduced capacity for muscle regeneration accompanied by an impaired proliferative potential of satellite cells that lead this capacity. Finally, the presence of protein aggregates in the muscle may overshoot protein clearance systems, such as proteasomal system, which contribute to muscle wasting and energy depletion. UPS, ubiquitin-proteasome system. 
glycinergic ones [56], compromising the functionality of the local SC motor circuitry. Indeed, alterations in inhibitory neurotransmission have been reported in ALS patients as well $[57,58]$, which extensively contribute to the imbalance between inhibitory and excitatory synaptic drive to MNs. Excitatory signaling gain and/or an inhibitory loss on MNs will increase $\mathrm{Ca}^{2+}$ influx and depolarization, favoring excitotoxicity $[59,60]$. It is not only a specific characteristic that happens in the SC but also in cortical areas. Functional studies, using transcranial magnetic stimulation of the motor cortex, showed an increase in the excitatory postsynaptic potentials recorded from the contralateral extensor digitorum communis muscle, a feature that Authors attribute to a marked loss of inhibition at cortical MNs in patients with ALS [61]. PET scanner studies [62] and in situ hybridization [63] revealed a decrease in both the density of the GABAA receptor and the expression of GABAA receptor subunit mRNA, respectively, in the motor cortex of ALS patients. Thus, all these evidences point to a loss of synaptic homeostasis in MNs, involving both lower and upper MNs and resulting from a decreased activity of the inhibitory circuits controlling MN excitability. Early alteration in cholinergic input from interneurons and output towards the NMJ and Renshaw cells concomitant to loss of inhibitory glycinergic synapses and Renshaw cells itself [64] may favor excitotoxicity definitely compromising regulatory microcircuitry function.

\section{GLIA CONTRIBUTION TO SYNAPTIC DYSFUNCTION IN ALS}

Astrocytes and microglia accompany MNs and react in consonance to neuronal function. The emerging concepts of the "tripartite" synapse [65], that includes astrocytes, and even the "quadripartite" synapse [66, 67] that add microglial cells as well, may better describe recent evidence indicating that there are multiple cell types working together at remodeling synaptic plasticity. One of the major functions of microglial cells in the brain is to survey for damage and protect neural material by orchestrating the innate immune response [68]. Microglia present an extremely cellular plasticity switching from a "resting" or "surveillant" cell phenotype with several fine processes to a globular "amoeboid" activated microglia with the ability to migrate to sites of damage and to phagocyte cellular debris [69]. Several evidences point to ramified "resting" microglia as highly dynamic surveyors of the healthy brain and determinant contributors to synaptic plasticity [70]. In particular, microglia contact with dendritic spines occur in a transient manner and linked to neuronal activity and functional experience as shown by Tremblay \& collaborators [71]. Several molecules that mediate microglia-neuron cross-talk have been related to these synaptic events including some cytokines with additional functions beyond their role in inflammation. One of these cytokines is tumor necrosis factor- $\alpha$ (TNF- $\alpha$ ), whose secretion has been recently specifically assigned to microglia but not to astrocytes [72]. Two transmembrane receptors, TNF receptor 1 (TNFR1) and TNFR2, were discovered 25 years ago [73]. TNFR1 is expressed ubiquitously on almost all cell types, while TNFR2 is more limited to specific cells, as those of the immune system [74]. However, activation of both TNFR1 and TNFR2 expression in neurons, astrocytes and oligodendrocytes was observed after traumatic CS injury [75]. While TNFR1 is predominantly linked to apoptosis and neurodegeneration, TNFR2 was shown to be involved in tissue regeneration and neuroprotection $[76,77]$.

However, TNF- $\alpha$ levels also control basal synaptic function and synaptic scaling plasticity [78]. Synaptic scaling is a form of non-associative homeostatic plasticity allowing equilibrium between excitatory and inhibitory inputs that works to stabilize neuronal firing rates by adjusting the strength of all the synapses of a neuron to keep firing rates roughly constant. TNF- $\alpha$ increases expression of surface AMPA receptors in both primary and slice cultures, hence increasing synaptic strength and synaptic scaling $[79,80]$. Both mRNA of TNF- $\alpha$ and its proapoptotic receptor TNFR1 were shown to be elevated in late presymptomatic stage and to increase progressively along disease progression in the lumbar $\mathrm{SC}$ of $\operatorname{tgSOD} 1^{\mathrm{G} 93 \mathrm{~A}}$ mice [81]. Interestingly, circulating levels of TNF- $\alpha$ and TNFR1 were found elevated in ALS patients, although no correlation with disease severity was not observed [82]. In ALS, the AMPA receptors presented an altered composition in subunit stoichiometry and editing that render them more permeable to calcium ions [83]. For this reason it has been considered a promising target and several drugs have been used to drive clinical trials although without success, probably because the excitotoxic phenomenon represents a too early phenomenon in disease onset. Besides the role of microglia on the plasticity of existing synapses it may also contribute to more profound structural changes 
by phagocyting specific subsets of synapses as it does during developmental synaptic pruning and in injury-related synaptic stripping events [84]. Moreover, as TNF- $\alpha$ is released by activated microglia one can consider neuroinflammation and excitotoxicity as mechanistic links for microglia-astrocyte and microglia-neuron cross-talk [85]. In these processes, other molecular axis of neuronal-microglia dialogue may also be implicated. Neuroprotective signaling from MN to microglia can be mediated through at least two cytokines, the chemokine ligand 1 (CX3CL1/fractalkine) and the membrane glycoprotein CD200 that have the correspondent receptors CX3CR1 and C200R, respectively, restricted to microglia [86, 87]. Increased expression of microglial C200R was found in the presymptomatic $\operatorname{tgSOD} 1^{\mathrm{G} 93 \mathrm{~A}}$ mice [88] while that of CX3CR1 was observed at disease endstage [89]. CX3CL1 signaling by healthy neurons controls the overproduction of iNOS, IL- $1 \beta$, TNF $\alpha$, and IL6 [90, 91]. In addition, CX3CR1 was also shown to play a role in cognitive function and synaptic plasticity [92]. Indeed, the axis has shown to have an important role in synaptic maturation and function since mice lacking CX3CR1 had deficits in synaptic pruning and decreased frequency of spontaneous excitatory post-synaptic currents, suggesting immature connectivity in the knockout mice [90]. In turn, microglial cathepsin S (CatS), a lysosomal/endosomal cysteine protease, seems to be responsible for the liberation of neuronal CX3CL1 [30]. Thus, CatS-mediated modification of the perisynaptic environment induce a downscaling of synaptic strength [93]. Information about the relevance of CX3CR1-CX3CL1 axis in ALS is scarce apart from that its disruption accelerates neurodegeneration in the double transgenic SOD $1^{\mathrm{G} 93 \mathrm{~A}} / \mathrm{Cx} 3 \mathrm{cr} 1-/-$ mice, which showed to survive less than the single SOD1 mutant [87].

Recent findings have underlined that toll-like receptor 2 (TLR2) and TLR4 are critical for glia activation. In the SC of ALS patients TLR2 was predominantly detected in microglia whereas TLR4 was strongly expressed in astrocytes [94]. Interestingly, while TLR2 evidenced to mediate inhibition of neural progenitor cell proliferation [95] and showed to be associated with synaptic loss, TLR4 was suggested to relate with synaptic stability in the SC after peripheral nerve injury [96]. In addition, microglial activation was also related with TDP-43 pathology and indicated to be an important determinant of cognitive impairment in ALS [97]. Finally, dystrophic microglia may have a determinant role to accelerate disease progression. As suggested by Graeber and Streit, chronic activation of microglial cells could lead to overactivation followed by microglial dystrophy and degeneration [98]. This is especially relevant in the context of aging-related disorders since microglia response to injury during early-life could increase the susceptibility to the development of disease in adulthood and aging [99]. Aged microglia present a decline in their dynamic motility through neural parenchyma, less frequent and extensive synaptic neuronal contacts, together with a decreased homeostatic regulation of neuronal activity and perturbations in the "on"/off signals, as well as increased neuroinflammatory response and oxidative stress [70]. The presence of mutant SOD1 acting on microglia is required to cause the disease [100] and its scission exclusively from microglia significantly slow disease progression [101]. Indeed, when the mutant SOD1 microglia was replaced with microglia expressing the wild-type SOD1 a prolonged survival of the ALS mice was observed [102]. Besides, the presence of mutant SOD1 intra- and extracellularly may enhance the production and release of reactive oxygen species (ROS), increase the inflammatory cytokine cascade [103] and accelerate the formation of the senescent microglial phenotype. Therefore, any neurotoxic effect that acts at a cellular level in ALS may affect the main functions of microglia promoting its early senescence and thus reducing synapse contacts, activity and plasticity that impede the possibility to regenerate disconnected MN.

\section{NEUROMUSCULAR COMPONENTS CONTRIBUTING TO SYNAPSE FAILURE IN ALS}

Cholinergic transmission towards the neuromuscular junction (NMJ) has also shown to be altered in ALS models. Decreased choline acetyltransferase (ChAT) expression and resultant reduction of acetylcholine (ACh) release from motor nerve ends can lead to dysfunction of motor synapses at axon terminals $[104,105]$. Impaired neuromuscular transmission was detected in early sALS patients [106], and a reduced supply of releasable ACh was thought to be the primary cause for the degeneration of motor terminals in a dog model of hereditary spinal muscular atrophy [104, 107]. Several factors can contribute to such issue. Recently, Tateno and collaborators reported an interesting hypothesis based on the alterations in axonal transport towards the NMJ terminal, 
in general, and to the transport of ChAT and $\mathrm{AChE}$ in particular. These molecules require binding to a motor protein to be transported along the axon, such as the kinesin-associated protein 3 (KAP3), which is specifically sequestered by the presence of misfolded SOD1 species. As a consequence, the transport of ChAT was shown to be interrupted at the pre-onset stage in the $\operatorname{tgSOD} 1^{\mathrm{G} 93 \mathrm{~A}}$ mice [108] and to accumulate in the MN soma [55]. Considering that misfolded SOD1 has been also linked to sALS, the inhibition of ChAT transport and hence the reduction in acetylcholine (ACh) availability may be one of the common pathways leading to $\mathrm{MN}$ specific dysfunction in ALS. ACh is necessary for the neurotransmission but also activates endplate receptors during spontaneous release. This may explain selective vulnerability observed between fast (more vulnerable) and slow MN types in ALS because fast MNs are more dependent on endplate ACh receptor activation during spontaneous release that acts then as a retrograde signaling system for regulating their electrical properties, maintaining connectivity and promoting regeneration [109]. Accordingly, Naumenko and collaborators have observed a significant decrease in the immediately available transmitter pool in nerve endings of tgSOD $1^{\mathrm{G} 93 \mathrm{~A}}$ mice males [110], likely one of the reasons for the dramatic drop in the spontaneous mediator release and enhanced fatigability of ALS muscles at high rate firing [111]. In this regard, a decrease in spontaneous ACh release would consistently weaken the strength of selective NMJ and hinder regeneration, as observed in ALS $[55,112]$.

Interestingly, it was recently evidenced that matrix metalloproteinase-9 (MMP-9) is expressed only by fast MNs and its pharmacological inhibition revealed to significantly delay muscle denervation [113]. MMP-9 activity was previously found enhanced in pyramidal neurons in the motor cortex and MNs in the SC of ALS patients [114], as well in their serum [115]. Moreover, other studies revealed that MMP-9 activity also increase in the SC and skin of the $\operatorname{tgSOD} 1^{\mathrm{G} 93 \mathrm{~A}}$ mouse model, mainly in the later stages of the disease [116]. Later, it was also shown that the extracellular media of differentiating NSC-34 MN-like cells carrying the G93A mutation in human SOD1 also contain increased MMP-9 activation [117]. Most important, these Authors demonstrated that MMP9 activation was reduced after treatment with the bile acid glycoursodeoxycolic acid, suggesting that it may be a promising therapeutic strategy when considering MMP-9 as a candidate target.NMJ fragility has long been considered a hallmark in ALS. Studies in rodents and humans have demonstrated that axonal retraction occurs well before the loss of cell bodies and that intrinsic axonal mechanisms actively contribute to phenotype in ALS [42, 118]. Actually, in the $\operatorname{tgSOD} 1^{\mathrm{G} 93 \mathrm{~A}}$ mouse model it was observed that denervation occurred as a very early phenomena, i.e. weeks before MN lost was noticed [119]. Such studies also demonstrated that fast-fatigable motor units presented higher susceptibility to degenerate than slow ones as mentioned $[118,120]$. Thus NMJ degeneration represent early pathological targets [112]. Is important to bear in mind that at the peripheral NMJ, three main different cell types, i.e. motor nerve terminal, terminal Schwann cells (TSC) and muscle fibers, collaborate in the assembly and maintenance of the synaptic apparatus. Non-myelinating, terminal Schwann cells (TSC), phenotypically different from those that wrap the axon in the peripheral nerve, cap the nerve terminal covering motor terminal branches and synaptic buttons and have a main role in regeneration [121]. A number of TSCs was noticed to be significantly reduced following disease progression in the $\operatorname{tgSOD} 1^{\mathrm{G} 93 \mathrm{~A}}$ rat muscle. In addition, it has been reported that TSCs specifically express the chemorepellent semaphorin 3A (Sema3A) at the fastfatigable-type neuromuscular synapses [122]. The authors propose that this de novo expression of Sema3A in the ALS rat model may impede nerve terminal plasticity at specific neuromuscular synapses, thus contributing to their early and selective loss.

Besides Sema 3A, other inhibitors for axonal regeneration such as Nogo-A have been observed originating from skeletal muscles, particularly from deltoid ALS patient biopsies and in tgSOD1 $193 \mathrm{~A}$ mice. This factor was proposed as a diagnostic [123] and prognostic [124] marker in ALS, once correlated with disease severity and motor impairment. Consistently, the double transgenic SOD $1^{\mathrm{G} 86 \mathrm{R}}$ and Nogo-A knock-out mice exhibited MN maintenance, reduced expression of denervation markers AChrna 1 alpha and Tyr kinase and prolonged lifespan [125]. However, the specificity of this factor in ALS disease remains controversial [126-128] as no increment of Nogo-A serum levels has been found in patients [129] and compensatory Nogo-B increment can underlay therapeutic benefits of Nogo-A knocking-out [130].

Failure or dysfunction of either the axonal metabolism itself or that of the skeletal muscle fibers contribute to this NMJ fragility and MN degeneration [131, 132]. The post-synaptic component of 
the NMJ, the muscle, plays an essential role in ALS axonopathy since overexpression of mutated SOD1 enzyme exclusively in skeletal muscle showed besides motor deficits such as spasticity and hyperreflexia, also abnormalities in the NMJ and a distal axonopathy $[133,134]$. However, apparent controversial results in the literature make difficult to achieve definite conclusions about muscle pathology contribution to ALS. In this regard, intramuscular injection of a lentiviral vector (not retrogradely transported to central nervous system), encoding a siRNA against SOD1 or specific gene excision through the CreLox-P recombinase system under creatine kinase promoter, showing a 50\% mRNA and protein reduction of mutant SOD1 exclusively in skeletal muscle provided no benefits of muscle grip strength maintenance and no delayed onset or progression of the disease [135]. However, due to the fact that even low the level of mutant SOD1 in skeletal muscle is able to trigger the ALS phenotype [133], reminiscent mutant SOD1 expression could be responsible for such observations. Several pathological events have been linked to muscle dysfunction in ALS and referred to influence the NMJ connection, such as metabolism and self-regenerative capacity. Dupuis and co-workers, described the up regulation of mitochondrial uncoupling protein (UCP) correlated with ATP reduction specifically in skeletal muscle of ALS patients and animal models [136]; they also demonstrated that muscle mitochondrial uncoupling driven by this protein leads to NMJ and MN dismantling, while exacerbating ALS phenotype [137]. In agreement, several Authors have reported increased lipid and carbohydrate clearance in tgSOD1 ${ }^{\mathrm{G} 93 \mathrm{~A}}$ and $\operatorname{tgSOD} 1{ }^{\mathrm{G} 86 \mathrm{R}}$ individual muscles at asymptomatic stages [138-140], correlating these findings with the muscle hypermetabolism and energy deficit. Consistently, high-fat diets have demonstrated to restore body mass, ameliorate muscle denervation and motor deficits and prolong the lifespan in animal models. Muscle mitochondrial defects have been largely described in skeletal muscle from ALS patients [138, 141, 142]. However, mitochondria are key intracellular organelles regulating not only energy but also other essential cellular functions, such as calcium homeostasis or apoptotic signaling. The expression of aberrant mutated proteins such as SOD1 in skeletal muscle myofibers of ALS models, as previously described in MNs, has been proposed to interfere with the inner membrane mitochondrial potential and the calcium buffering capacity [143] that results in the sequestrate of the anti-apoptotic protein Bcl-2, lead- ing to apoptosis and development of local oxidative stress [144, 145]. Interestingly, Wei R and colleagues did not detect the presence of mutant protein aggregates in gastrocnemius muscle of $\operatorname{tgSOD} 1^{\mathrm{G} 93 \mathrm{~A}}$ mice from pre-symptomatic (50 days) to terminal stages (130 days), although a 2 to 4 fold increase in $20 \mathrm{~S}$ proteasomal activity was observed. These Authors concluded that the muscle ability to maintain a high proteasomal activity mediated the effective clearance of the toxic protein aggregates [146] as it had been previously suggested in C2C12 myoblasts [147]. The ubiquitin-proteasome system (UPS) is crucial for maintaining muscle homeostasis, as it is required to remove sarcomeric proteins upon changes in muscle activity [148, 149]; excessive up-regulation through increased proteasomal activity, ubiquitin-conjugation or over-expression of transcripts encoding ubiquitin, ubiquitin-conjugating enzymes, ubiquitin-protein ligases or proteasomal subunits, leads to muscle wasting [150]. Importantly, myofiber atrophy is one of the earliest events on ALS. Studies with specific modulators of this pathway would be of interest to establish the optimal UPS activity beneficial for mutant protein clearance but not detrimental for the maintenance of muscle mass on ALS.

Molecular signaling alterations have been also found in RNA processing both mRNA and miRNA. mRNA processing was found disturbed in ALS mouse models; consistently, oxidized mRNA species were detected in the SC of patients, as well as in $\operatorname{tgSOD} 1^{\mathrm{G} 93 \mathrm{~A}}$ mice at the pre-symptomatic 45 days [151]. Tight implication of miRNA biogenesis and performance in muscle cells is likely to operate on ALS since miRNA 23a, 455, 29b and 206 were upregulated in vastus lateralis of ALS patients [152]. Both miRNA 29 and 206 are known to promote muscle regeneration and recovery after cardiotoxin or nerve crush injury $[153,154]$ and ALS mouse model genetically deficient in miR-206 showed accelerated progression of the disease [155].

In adults, satellite cell pool, largely responsible for the regenerative capacity in this tissue, remain quiescent but retain the ability to activate and reenter cell cycle in response to signals from the cellular niche such as muscle injury or intense physical exercise. A portion of these cells proliferate and fuse with each other or with the parental myofiber to fully regenerate the damaged tissue, whereas the rest remain undifferentiated and exit cell cycle to replenish the myogenic cell pool. This process is regulated by highly coordinated transcriptional and translational networks in which myogenic regula- 
tory factors play a central role [156-160]. However, in aging [161, 162], Duchenne muscular dystrophy $[163,164]$ or spinal muscular atrophy [165], successive rounds of regeneration or aberrant stimuli from the cellular niche perturb self-renewal or myogenesis leading to depletion of the satellite cell pool or loss of myogenic potential and muscle wasting. In line with this, it has been recently reported an abnormal number of satellite cells in both fast and slowtwitch myofibers of $\operatorname{tgSOD} 1^{\mathrm{G} 93 \mathrm{~A}}$ mouse model since the earliest pre-symptomatic stages of the disease, accompanied by blunted protein translation of myogenic regulatory factors controlling the activity of these cells and in vitro impaired proliferative potential [166].

Despite the uncertain mechanisms of $\mathrm{MN}$ degeneration driven by the skeletal muscle, several evidences exist based on therapeutic benefits proportionated by strategies supporting muscle homeostasis, regenerative potential and muscle mass. Controlled exercise improved motor function and survival in $\operatorname{tgSOD} 1^{\mathrm{G} 93 \mathrm{~A}}$ animal models [167] and reduced spasticity and motor function loss in ALS patients [168]. Mild physical activity has neuroprotective benefits as promotes neurogenesis [168-170] and increases peripheral levels of neurotrophic factors such as BDNF (brain-derived neurotrophic factor) [171-173] and IGF-1 [174, 175]. However, muscle training is an inductor of oxidative stress, as markedly increases the systemic consumption and uptake of oxygen thus enhancing ROS production, including the generation of the superoxide radical from the mitochondrial electron transport chain [176]. Therefore, despite substantial effort to establish the optimal stage, intensity and duration of the training to maximize the potential benefits, the controversy remains [174, 177-179]. These observations, together with the high incidence of ALS cases within professionals developing intense physical activity, such as soccer players [180-183], encouraged clinicians to advice against such practice to preserve muscle mass.

Artificial delivery of neurotrophic molecules, such as muscle-derived but no centrally derived GDNF (glial-derived neurotrophic factor) over-expression, was neuroprotective in the $\operatorname{tgSOD} 1^{\mathrm{G} 93 \mathrm{~A}}$ mice [184]. Indeed, intramuscular injections of myoblasts [185] and mesenchymal stem cells [186] carrying vectors encoding GDNF, expanded the survival and motor performance of $\operatorname{tgSOD} 1^{\mathrm{G} 93 \mathrm{~A}}$ rodents and delayed MN loss, as shown by levels of acetylcholine receptor postsynaptic clusters and ChAT or agrin-positive end plates within the lumbar SC region. Additionally, IGF-1 restricted expression in the skeletal muscle of ALS rodents counteracted the symptoms of the disease, exacerbated myogenesis and reduced catabolism, thus prolonging the lifespan [21, 187, 188]. These Authors concluded that skeletal muscle satellite cell activity, mediated by muscle IGF-1, contributed to the ameliorated phenotype of the $\operatorname{tgSOD} 1^{\mathrm{G} 93 \mathrm{~A}}$ mice.

Overall these findings provide evidence that ALS skeletal muscle suffer from direct neurotoxic effects of SOD1 or other molecules and definitely affect synaptic connectivity at the NMJ, as well as its metabolism, contributing to the multi-systemic phenotype of the disease.

\section{MN PATHOLOGY AND CONTRIBUTION TO SYNAPTIC DYSFUNCTION IN ALS}

Several years ago, some Authors suggested that synaptic dysfunction may precede synaptic loss [51, $189,190]$ and, indeed, this is a rather common feature in other neurodegenerative diseases as well [191]. In both sALS and fALS, molecular changes most likely precede the clinical onset. In sALS, an acquired defect in chromatin, DNA, RNA or proteins (such as epigenetic alteration, a somatic DNA mutation, a RNA editing error, and the misfolding of proteins possibly due to ageing effects or environmental stressors) occurs randomly in each cell, and these defects accumulate and converge to initiate the pathogenic process. The mechanisms involved in $\mathrm{MN}$ dysfunction in ALS have been accurately detailed recently by Cozzolino and collaborators [192]. The discovery of new genes implicated in ALS and the existence of common mechanisms found in most of the genes have pointed to new insights into the pathogenic pathways. Bioinformatics' analysis of their annotated functions reveal an enrichment in processes such as endocytosis and impaired vesicle (Alsin, VAPB, OPTN, VCP), axonal transport and organelle trafficking (spatacsin), compromised autophagy $(C H M P 2 B)$ and protein degradation, in the UPR response (UBQLN2, SIGMAR1), transcription regulation and RNA metabolism (C9orf72, TARDBP, $A N G, S E T X)$, as well as in mitochondria-dependent oxidative stress. Increased levels of "classical" markers of oxidative stress, including reactive oxygen species (ROS) and products of protein and lipid oxidation, have been repeatedly and consistently observed in tissues from patients and in in vitro and 
in vivo mutant SOD1 models, although its origin is still not clear [193]. Since, mitochondria are the major sites of formation of ROS and are particularly susceptible to oxidative stress, the vicious cycle can pushover mitochondrial damage. Besides, connectivity among different organelles reveal some key joined events related to pathogenesis in ALS. As mentioned by Cozzolino and collaborators in their review [192], the interaction between mitochondria and endoplasmic reticulum (ER), termed the ER-mitochondria calcium cycle (ERMCC), couples energy metabolism in mitochondria and protein processing in the ER to the synaptic activity in neurons mediated by AMPA receptor. Therefore, ERMCC seems to mediate AMPA receptor-mediated glutamate excitotoxicity in MNs where a toxic shift of calcium from the ER compartment to the mitochondrial compartment causes chronic calcium depletion of the ER and chronic mitochondrial calcium overload. The former would trigger the protective unfolded protein response (UPR), and the latter, a consecutive release of cell death signals through the mitochondrial permeability transition pore. Persistent ERMCC dysregulation is a key pathway resulting in the accumulation of misfolded proteins, failing UPR and MN death. The failure of protein homeostatic handling in the cell have been revealed by reported defects in UPR, in protective autophagy and in adequate protein quality control in ALS models [194, 195], as well as in SC tissues of human sALS patients [196]. But importantly, SOD1, OPTN, FUS/TLS \& TDP43 are prone to aggregate when mutated, but even in sALS, SOD1 aggregation represents a common hallmark [197]. It has been indicated that the increased aggregation propensity of SOD1 mutants is related to the decrease survival in ALS patients, further suggesting that aggregation could be a major contributor to SOD1 toxicity in such patients [198]. Importantly, several animal models with reduced motor function presented also impaired autophagy. Conceivable, motor defects generate high amounts of stranded cargo that may overwhelm the autophagic apparatus. Besides, some reports implicate these proteins also in synaptic function. TDP43 and FUS/TLS form part of the so-called mRNA-silencing foci that are cytoplasmatic supramolecular aggregates of repressed RNA and inhibitory proteins in charge of local translation induced specifically by synaptic activity in dendrites and synapses [199], while Optineurin is involved in vesicle trafficking needed for synapse maintenance [200]. The assignment of apoptosis to the ended mechanism of neuronal death in ALS has been for a subject of extensive debate since controversial evidences have been obtained when human samples were analyzed and matched with effects obtained in animal models [201-204]. What seems clear, however, is that if apoptosis exists it occurs only as a later event of a degenerating process that follows a "Dying Back" pattern of MN dysfunction [192].

Analysis of transcriptomic profile in neurodegenerative diseases has advanced considerably in the past 5 years. Increased scientific rigor and improved analytical tools have led to more-reproducible data. Many platforms for transcriptome analysis assay the expression of the entire genome, enabling that a complete biological context is captured. Gene expression profiling pointed to dysregulation of genes related to neuroinflammation, as well as to disruption of RNA splicing and protein turnover, and several studies in ALS also support the cytoskeleton involvement. Certainly, results from omics experiments will contribute to have a more integrative vision in the pathology of MNs and in its interaction with the cellular neighbors. Computational biology will help systems biology analysis to definitely ascertain the optimal combination of therapeutic agents to end up with the neurodegenerative process. This so-called network medicine [205] has been advanced fruitfully nowadays in cancer research and is certainly the next frontier also for complex neurodegenerative diseases such as ALS.

\section{CONCLUSION}

Consideration of ALS as a type of MN neuron disease has evolved to be considered as a multi-systemic, non-cell autonomous and complex neurodegenerative disease. Dying forward and dying back hypothesis to explain the etiology of $\mathrm{MN}$ degeneration are difficult to demonstrate in experimental models since rodent corticospinal circuitry differs substantially from that of humans. In that regard, it would be more plausible that noxa implicated in degeneration would affect specifically and importantly MNs mainly, independently on their location, favoring the hypothesis that complex genetic-environmental interaction is indeed the causal factor for $\mathrm{MN}$ degeneration. Histological and functional analysis on microcircuitry at the SC, brain and neuromuscular levels where MNs, interneurons, glial and muscle cells may interplay already revealed marked synaptic alterations that may compromise tis- 
sular homeostasis and that extensively will contribute to the imbalance between inhibitory and excitatory synaptic drive to MNs. Excitatory signaling gain and/or an inhibitory loss on MNs will increase $\mathrm{Ca}^{2+}$ influx and depolarization, favoring excitotoxicity largely observed in several regions of the CNS in patients and models. Besides, particular early affectation on cholinergic transmission within the CNS and towards the NMJ might contribute to synaptic dysfunction first, followed by later cellular dysfunction and even selective MN degeneration. Since glial cells, particularly microglia at the CNS and terminal Schwann cells at the NMJ, contribute to synaptic remodeling and maintenance, it will be worth focus the attention on molecules involved in crosstalking with neurons and axons, as well as in aspects regarding the effects of glial senescence in disease environment and its repercussions on neuronal function failure. Finally, special emphasis on metabolism pitfalls present in ALS patients shifted the attention to muscle regenerative capabilities in patients and models, which have been observed as being severely affected. ALS skeletal muscle suffers from direct neurotoxic effects of SOD1, or other molecules, which definitely affect synaptic connectivity at the NMJ, as well as its metabolism, contributing to the multisystemic phenotype of the disease. With all these landscape together with intrinsic abnormalities, MN function evolve towards dysfunction of its main cellular processes related to transcription, translation and vesicle trafficking, considered to be fundamental in synapse maintenance, connectivity and finally in survival.

\section{ACKNOWLEDGMENTS}

This work was supported by grants from Fundació LaMarató-TV3 (110430/31/32) and TERCEL and CIBERNED funds from the Fondo de Investigación Sanitaria of Spain, by FEDER (COMPETE Programme) and Portugal National funds (FCT Fundação para a Ciência e a Tecnologia - -project PTDC/SAU-FAR/118787/2010 to DB and PEstOE/SAU/UI4013/2011-14 to iMed.ULisboa). ARV is recipient of a Post-Doctoral fellowship (FCT SFRH/BPD/76590/2011). Authors declare no conflict of interest.

\section{REFERENCES}

[1] Charcot J, Joffroy A (1869) Deux cas d'atrophie musculaire progressive avec lesions de la substance grise et des faisceaux antero-lateraux de la moelle epiniere. Arch Physiol Norm path 2, 744-760.

[2] Kumar DR, et al. (2011) Jean-martin charcot: the father of neurology. Clin Med Res 9, 46-49.

[3] Meininger V (2011) ALS, what new 144 years after Charcot? Arch Ital Biol 149, 29-37.

[4] Brain W, Walton J (1969) Brain's diseases of the nervous system, London, Oxford University Press.

[5] Gordon PH (2013) Amyotrophic lateral sclerosis: an update for 2013 clinical features, pathophysiology, management and therapeutic trials, Aging Dis 4, 295-310.

[6] Chiò A, et al. (2013) Global epidemiology of amyotrophic lateral sclerosis: a systematic review of the published literature. Neuroepidemiology 41, 118-130.

[7] Chio A, et al. (2009) Prognostic factors in ALS: a critical review. 10, 310-323.

[8] Wijesekera LC, Leigh PN (2009) Amyotrophic lateral sclerosis. Orphanet J Rare Dis 4, 3.

[9] Kiernan MC, et al. (2011) Amyotrophic lateral sclerosis. Lancet 377, 942-955.

[10] Turner MR, et al. (2013) Controversies and priorities in amyotrophic lateral sclerosis. Lancet Neurol 12, 310-322.

[11] Dupuis L, et al. (2011) Energy metabolism in amyotrophic lateral sclerosis. Lancet Neurol 10, 75-82.

[12] Garbuzova-Davis S, et al. (2007) Ultrastructure of blood-brain barrier and blood-spinal cord barrier in SOD1 mice modeling ALS. Brain Res 1157, 126-137.

[13] Brites D, et al. (2012) Neurovascular unit: a focus on pericytes. Molecular Neurobiology 45 (2012), 327-347.

[14] Andjus PR, et al. (2009) In vivo morphological changes in animal models of amyotrophic lateral sclerosis and Alzheimer's-like disease: MRI approach. Anat Rec (Hoboken) 292, 1882-1892.

[15] Nicaise C, et al. (2009) Impaired blood-brain and bloodspinal cord barriers in mutant SOD1-linked ALS rat. Brain Res 1301, 152-162.

[16] Zhong Z, et al. (2008) ALS-causing SOD1 mutants generate vascular changes prior to motor neuron degeneration. Nat Neurosci 11, 420-422.

[17] Jaarsma D, et al. (2008) Neuron-specific expression of mutant superoxide dismutase is sufficient to induce amyotrophic lateral sclerosis in transgenic mice. J Neurosci 28, 2075-2088.

[18] Philips T, Robberecht W (2011) Neuroinflammation in amyotrophic lateral sclerosis: role of glial activation in motor neuron disease. Lancet Neurol 10, 253-263.

[19] Liao B, et al. (2012) Transformation from a neuroprotective to a neurotoxic microglial phenotype in a mouse model of ALS. Exp Neurol 237, 147-152.

[20] Kang SH, et al. (2013) Degeneration and impaired regeneration of gray matter oligodendrocytes in amyotrophic lateral sclerosis. Nat Neurosci. DOI: 10.1038/nn.3357

[21] Dobrowolny G, et al. (2008) Skeletal muscle is a primary target of SOD1G93A-mediated toxicity. Cell Metab 8, 425-436.

[22] Czirr E, Wyss-Coray T (2012) The immunology of neurodegeneration. J Clin Invest 122, 1156-1163.

[23] Liblau RS, et al. (2013) Neurons as targets for T cells in the nervous system. Trends Neurosci 36, 315-324.

[24] Winkler EA, et al. (2013) Blood-spinal cord barrier breakdown and pericyte reductions in amyotrophic lateral sclerosis. Acta Neuropathol 125, 111-120.

[25] Turner MR, Kiernan MC (2012) Does interneuronal dysfunction contribute to neurodegeneration in amyotrophic lateral sclerosis? Amyotroph Lateral Scler 13, 245-250. 
[26] Deng HX, et al. (1993) Amyotrophic lateral sclerosis and structural defects in $\mathrm{Cu}, \mathrm{Zn}$ superoxide dismutase. Science 261, 1047-1051.

[27] Lattante S, et al. (2013) TARDBP and FUS mutations associated with amyotrophic lateral sclerosis: summary and update. Hum Mutat 34, 812-826.

[28] Wuolikainen A, et al. (2012) ALS patients with mutations in the SOD1 gene have an unique metabolomic profile in the cerebrospinal fluid compared with ALS patients without mutations. Mol Genet Metab 105, 472-478.

[29] Berthod F, Gros-Louis F. (2012) In vivo and in vitro models to study amyotrophic lateral sclerosis. Amyotroph Lateral Sclerosis Intech 3

[30] Brites D, Vaz AR. (2014) Microglia centered pathogenesis in ALS: insights in cell interconnectivity. Front Cell Neurosci 8, 117.

[31] Poindron P, Borg J. (1997) Progressive motor neuron impairment in an animal model of familial amyotrophic lateral sclerosis.

[32] Bendotti C, Carri MT. (2004) Lessons from models of SOD1-linked familial ALS. 10.

[33] Battistini S, et al. (2005) SOD1 mutations in amyotrophic lateral sclerosis: results from a multicenter Italian study. J Neurol 252, 782-788.

[34] Bories C, et al. (2007) Early electrophysiological abnormalities in lumbar motoneurons in a transgenic mouse model of amyotrophic lateral sclerosis. 25, 451-459.

[35] Amendola J, et al. (2004) Short communication Altered sensorimotor development in a transgenic mouse model of amyotrophic lateral sclerosis. 20, 2822-2826.

[36] Chen S, et al. (2013) Genetics of amyotrophic lateral sclerosis: an update. Mol Neurodegener 8, 28.

[37] Hardy J, Rogaeva E. (2013) Motor neuron disease and frontotemporal dementia: sometimes related, sometimes not. Exp Neurol. DOI: 10.1016/j.expneurol.2013.11.006

[38] Yang C, et al. (2014) Partial loss of TDP-43 function causes phenotypes of amyotrophic lateral sclerosis. Proc Natl Acad Sci U S A 111, E1121-E1129.

[39] Rojas F, et al. (2014) Astrocytes expressing mutant SOD1 and TDP43 trigger motoneuron death that is mediated via sodium channels and nitroxidative stress. Front Cell Neurosci 8, 24.

[40] Feneberg E, et al. (2014) Limited role of free TDP43 as a diagnostic tool in neurodegenerative diseases. Amyotroph Lateral Scler Frontotemporal Degener. DOI: 10.3109/21678421.2014.905606

[41] Mackenzie IRA, et al. (2014) The neuropathology associated with repeat expansions in the C9ORF72 gene. Acta Neuropathologica 127, 347-357.

[42] Fischer LR, et al. (2004) Amyotrophic lateral sclerosis is a distal axonopathy: evidence in mice and man. Exp Neurol 185, 232-240.

[43] Kiernan JA, Hudson AJ (1991) Changes in sizes of cortical and lower motor neurons in amyotrophic lateral sclerosis. Brain 114, 843-853.

[44] Münch C, et al. (2011) Prion-like propagation of mutant superoxide dismutase-1 misfolding in neuronal cells. Proc Natl Acad Sci U S A 108, 3548-3553.

[45] Grad LI, et al. (2011) Intermolecular transmission of superoxide dismutase 1 misfolding in living cells. Proc Natl Acad Sci U S A 108, 16398-16403.

[46] Kanouchi T, et al. (2012) Can regional spreading of amyotrophic lateral sclerosis motor symptoms be explained by prion-like propagation? J Neurol Neurosurg Psychiatry 83, 739-745.
[47] Bareyre FM, et al. (2005) Transgenic labeling of the corticospinal tract for monitoring axonal responses to spinal cord injury. Nat Med 11, 1355-1360.

[48] Kiryu-Seo S, et al. (2005) Noxa is a critical mediator of p53-dependent motor neuron death after nerve injury in adult mouse. J Neurosci 25, 1442-1447.

[49] Seo YW, et al. (2009) The cell death-inducing activity of the peptide containing noxa mitochondrial-targeting domain is associated with calcium release. Cancer Res 69, 8356-8365.

[50] Ikemoto A, et al. (1994) Immunocytochemical studies on synaptophysin in the anterior horn of lower motor neuron disease. J Neuropathol Exp Neurol 53, 196-201.

[51] Sasaki S, Maruyama S (1994) Decreased synaptophysin immunoreactivity of the anterior horns in motor neuron disease. Acta Neuropathol 87, 125-128.

[52] Ikemoto A, et al. (2002) Differential expression between synaptic vesicle proteins and presynaptic plasma membrane proteins in the anterior horn of amyotrophic lateral sclerosis. Acta Neuropathol 103, 179-187.

[53] Zang DW, et al. (2005) Loss of synaptophysin-positive boutons on lumbar motor neurons innervating the medial gastrocnemius muscle of the SOD1G93A G1H transgenic mouse model of ALS. J Neurosci Res 79, 694-699.

[54] Nagao M, et al. (1998) Loss of cholinergic synapses on the spinal motor neurons of amyotrophic lateral sclerosis. J Neuropathol Exp Neurol 57, 329-333.

[55] Casas C, et al. (2013) Early presymptomatic cholinergic dysfunction in a murine model of amyotrophic lateral sclerosis. Brain Behav 3, 145-158.

[56] Chang Q, Martin LJ (2009) Glycinergic innervation of motoneurons is deficient in amyotrophic lateral sclerosis mice: a quantitative confocal analysis. Am J Pathol 174, 574-585.

[57] Hayashi H, et al. (1981) Reduced glycine receptor in the spinal cord in amyotrophic lateral sclerosis. Ann Neurol 9 , 292-294.

[58] Whitehouse PJ, et al. (1983) Amyotrophic lateral sclerosis: alterations in neurotransmitter receptors. Ann Neurol 14, 8-16.

[59] Sunico CR, et al. (2011) Reduction in the motoneuron inhibitory/excitatory synaptic ratio in an earlysymptomatic mouse model of amyotrophic lateral sclerosis. Brain Pathol 21, 1-15.

[60] Schutz B (2005) Imbalanced excitatory to inhibitory synaptic input precedes motor neuron degeneration in an animal model of amyotrophic lateral sclerosis. Neurobiol Dis 20, 131-140.

[61] Enterzari-Taher M, et al. (1997) Abnormalities of cortical inhibitory neurons in amyotrophic lateral sclerosis. Muscle Nerve 20, 65-71.

[62] Lloyd CM, et al. (2000) Extramotor involvement in ALS: PET studies with the GABA(A) ligand [(11)C]flumazenil. Brain 123(Pt 1), 2289-2296.

[63] Petri S, et al. (2003) Distribution of GABAA receptor mRNA in the motor cortex of ALS patients. J Neuropathol Exp Neurol 62, 1041-1051.

[64] Wootz H, et al. (2013) Alterations in the motor neuronrenshaw cell circuit in the Sod1(G93A) mouse model. J Comp Neurol 521, 1449-1469.

[65] Araque A, et al. (1999) Tripartite synapses: glia, the unacknowledged partner. Trends Neurosci 22, 208-215.

[66] Schafer DP, et al. (2013) The "quad-partite" synapse: microglia-synapse interactions in the developing and mature CNS. Glia 61, 24-36. 
[67] Tremblay MÈ, Majewska AK (2011) A role for microglia in synaptic plasticity? Commun Integr Biol 4, 220-222.

[68] Rivest S (2009) Regulation of innate immune responses in the brain. Nat Rev Immunol 9, 429-439.

[69] Morris GP, et al. (2013) Microglia: a new frontier for synaptic plasticity, learning and memory, and neurodegenerative disease research. Neurobiol Learn Mem 105, 40-53.

[70] Wong WT. (2013) Microglial aging in the healthy CNS: phenotypes, drivers, and rejuvenation. Front Cell Neurosci $7,22$.

[71] Tremblay M-È, et al. (2010) Microglial interactions with synapses are modulated by visual experience. PLOS Biol 8,16 .

[72] Béchade C, et al. (2013) Microglial control of neuronal activity. Front Cell Neurosci 7, 32.

[73] Hohmann HP, et al. (1989) Two different cell types have different major receptors for human tumor necrosis factor (TNF alpha). J Biol Chem 264, 14927-14934.

[74] Naudé PJW, et al. (2011) Tumor necrosis factor receptor cross-talk. FEBS J 278, 888-898.

[75] Yan P. (2003) Expression of the type 1 and type 2 receptors for tumor necrosis factor after traumatic spinal cord injury in adult rats. Experimental Neurology 183, 286-297.

[76] Venters HD, et al. (2000) A new concept in neurodegeneration: TNFalpha is a silencer of survival signals. Trends Neurosci 23, 175-180.

[77] Fischer R, et al. (2011) A TNF receptor 2 selective agonist rescues human neurons from oxidative stress-induced cell death. PLoS One 6.

[78] Santello M, et al. (2011) TNF[alpha] controls glutamatergic gliotransmission in the hippocampal dentate gyrus. Neuron 69, 988-1001.

[79] Stellwagen D, Malenka RC (2006) Synaptic scaling mediated by glial TNF-alpha. Nature 440, 1054-1059.

[80] Ben Achour S, Pascual O (2010) Glia: the many ways to modulate synaptic plasticity. Neurochem. Int $\mathbf{5 7}$, 440-445.

[81] Hensley K, et al. (2002) Temporal patterns of cytokine and apoptosis-related gene expression in spinal cords of the G93A-SOD1 mouse model of amyotrophic lateral sclerosis.

[82] Poloni M, et al. (2000) Circulating levels of tumour necrosis factor-alpha and its soluble receptors are increased in the blood of patients with amyotrophic lateral sclerosis. Neurosci Lett 287, 211-214.

[83] Hideyama T, et al. (2012) Profound downregulation of the RNA editing enzyme ADAR2 in ALS spinal motor neurons. Neurobiol Dis 45, 1121-1128.

[84] Cullheim S, Thams S (2007) The microglial networks of the brain and their role in neuronal network plasticity after lesion. Brain Res Rev 55, 89-96.

[85] Olmos G, Lladó J. (2014) Tumor necrosis factor alpha: a link between neuroinflammation and excitotoxicity. Mediators of Inflammation 2014.

[86] Hoek RM, et al. (2000) Down-regulation of the macrophage lineage through interaction with OX2 (CD200). Science 290, 1768-1771.

[87] Cardona AE, et al. (2006) Control of microglial neurotoxicity by the fractalkine receptor. Nat Neurosci 9, 917-924.

[88] Chen L-C, et al. (2004) Temporal gene expression patterns in G93A/SOD1 mouse. Amyotroph Lateral Scler Other Motor Neuron Disord 5, 164-171.

[89] Lewis C-AB, et al. (2009) Bone marrow-derived cells in the central nervous system of a mouse model of amy- otrophic lateral sclerosis are associated with blood vessels and express CX(3)CR1. Glia 57, 1410-1419.

[90] Bachstetter AD, et al. CX3CR1 deficiency leads to impairment of hippocampal cognitive function and synaptic plasticity. Journal of Neuroscience 31 (2011), 1624116250.

[91] Biber K, et al. (2007) Neuronal "On" and "Off" signals control microglia. Trends in Neurosciences 30, 596-602.

[92] Rogers JT, et al. CX3CR1 deficiency leads to impairment of hippocampal cognitive function and synaptic plasticity. Journal of Neuroscience 31 (2011), 16241-16250.

[93] Hayashi Y, et al. (2013) The intrinsic microglial molecular clock controls synaptic strength via the circadian expression of cathepsin S. Sci Rep 3, 2744.

[94] Casula M, et al. (2011) Toll-like receptor signaling in amyotrophic lateral sclerosis spinal cord tissue. Neuroscience 179, 233-243.

[95] Okun E, et al. (2010) TLR2 activation inhibits embryonic neural progenitor cell proliferation. J Neurochem 114, 462-474.

[96] Freria CM, et al. (2012) Opposing effects of Toll-like receptors 2 and 4 on synaptic stability in the spinal cord after peripheral nerve injury. J Neuroinflammation $\mathbf{9}$, 240.

[97] Brettschneider J, et al. (2012) Microglial activation and TDP-43 pathology correlate with executive dysfunction in amyotrophic lateral sclerosis. Acta Neuropathol 123, 395-407.

[98] Graeber MB, Streit WJ (2010) Microglia: biology and pathology. Acta Neuropathol 119, 89-105.

[99] Somera-Molina KC, et al. (2009) Enhanced microglial activation and proinflammatory cytokine upregulation are linked to increased susceptibility to seizures and neurologic injury in a "two-hit" seizure model. Brain Res 1282, 162-172.

[100] Clement AM, et al. (2003) Wild-type nonneuronal cells extend survival of SOD1 mutant motor neurons in ALS mice. Science 302, 113-117.

[101] Boillée S, et al. (2006) Onset and progression in inherited ALS determined by motor neurons and microglia. Science 312, 1389-1392.

[102] Lee JC, et al. (2012) Replacement of microglial cells using Clodronate liposome and bone marrow transplantation in the central nervous system of SOD1(G93A) transgenic mice as an in vivo model of amyotrophic lateral sclerosis. Biochem Biophys Res Commun 418, 359-365.

[103] Henkel JS, et al. (2009) Microglia in ALS: the good, the bad, and the resting. J Neuroimmune Pharmacol 4, 389398.

[104] Rich MM, et al. (2002) Reduced neuromuscular quantal content with normal synaptic release time course and depression in canine motor neuron disease. J Neurophysiol 88, 3305-3314

[105] Brandon EP, et al. (2003) Aberrant patterning of neuromuscular synapses in choline acetyltransferase-deficient mice. J Neurosci 23, 539-549.

[106] Maselli RA, et al. (1993) Neuromuscular transmission in amyotrophic lateral sclerosis. Muscle Nerve 16, 11931203.

[107] Balice-Gordon RJ, et al. (2000) Functional motor unit failure precedes neuromuscular degeneration in canine motor neuron disease. Ann Neurol 47, 596-605.

[108] Tateno M, et al. (2009) Mutant SOD1 impairs axonal transport of choline acetyltransferase and acetylcholine release by sequestering KAP3. Hum Mol Genet 18, 942-955. 
[109] Reid B, et al. (1999) Synaptic vesicle dynamics in rat fast and slow motor nerve terminals. J Neurosci 19, 2511-2521.

[110] Naumenko N, et al. (2011) Gender-specific mechanism of synaptic impairment and its prevention by GCSF in a mouse model of ALS. Front Cell Neurosci 5, 26.

[111] Schomburg ED, et al. (2011) Fatigability of spinal reflex transmission in a mouse model (SOD1(G93A)) of amyotrophic lateral sclerosis. Muscle Nerve 43, 230-236.

[112] Murray LM, et al. (2010) Review: Neuromuscular synaptic vulnerability in motor neurone disease: amyotrophic lateral sclerosis and spinal muscular atrophy. Neuropathol Appl Neurobiol 36, 133-156.

[113] Kaplan A, et al. (2014) Neuronal matrix Metalloproteinase-9 is a determinant of selective Neurodegeneration. Neuron 81, 333-348.

[114] Lim GP, et al. (1996) Matrix metalloproteinases in the neocortex and spinal cord of amyotrophic lateral sclerosis patients. J Neurochem 67, 251-259.

[115] Beuche W, et al. (2000) Matrix metalloproteinase-9 is elevated in serum of patients with amyotrophic lateral sclerosis. Neuroreport 11, 3419-3422.

[116] Fang L, et al. (2010) MMP-2 and MMP-9 are elevated in spinal cord and skin in a mouse model of ALS. J Neurol Sci 294, 51-56.

[117] Vaz AR, et al. (2014), Glycoursodeoxycholic acid reduces matrix metalloproteinase- 9 and caspase- 9 activation in a cellular model of superoxide dismutase- 1 neurodegeneration. Molecular Neurobiology.

[118] Frey D, et al. (2000) Early and selective loss of neuromuscular synapse subtypes with low sprouting competence in motoneuron diseases. J Neurosci 20, 2534-2542.

[119] Pun S, et al. (2006) Selective vulnerability and pruning of phasic motoneuron axons in motoneuron disease alleviated by CNTF. Nat Neurosci $9,408-419$.

[120] Kennel PF, et al. (1996) Neuromuscular function impairment is not caused by motor neurone loss in FALS mice: an electromyographic study. Neuroreport 7, 1427-1431.

[121] Reddy LV, et al. (2003) Glial cells maintain synaptic structure and function and promote development of the neuromuscular junction in vivo. Neuron 40, 563-580.

[122] De Winter F, et al. (2006) The expression of the chemorepellent Semaphorin $3 \mathrm{~A}$ is selectively induced in terminal Schwann cells of a subset of neuromuscular synapses that display limited anatomical plasticity and enhanced vulnerability in motor neuron disease. Mol Cell Neurosci 32, 102-117.

[123] Dupuis L, et al. (2002) Nogo provides a molecular marker for diagnosis of amyotrophic lateral sclerosis. Neurobiol Dis 10, 358-365.

[124] Jokic N, et al. (2005) Nogo expression in muscle correlates with amyotrophic lateral sclerosis severity. Ann Neurol 57, 553-556.

[125] Jokic N, et al. (2006) The neurite outgrowth inhibitor Nogo-A promotes denervation in an amyotrophic lateral sclerosis model. EMBO Rep 7, 1162-1167.

[126] Calvo AC, et al. (2012) Genetic biomarkers for ALS disease in transgenic SOD1G93A mice. PLoS ONE 7, e32632.

[127] Sun A, et al. (2011) Significance of Nogo-A expression of atrophic muscle fibers in diagnosis of amyotrophic lateral sclerosis. Beijing Da Хие Хие Bao 43, 238-241.

[128] Wojcik S, et al. (2006) Increased expression of Noga-A in ALS muscle biopsies is not unique for this disease. Acta Myol 25, 116-118.
[129] Harel NY, et al. (2009) Serum Nogo-A levels are not elevated in amyotrophic lateral sclerosis patients. Biomarkers 14, 414-417.

[130] Yang YS, et al. (2009) Reticulon-4A (Nogo-A) redistributes protein disulfide isomerase to protect mice from SOD1-dependent amyotrophic lateral sclerosis. $\mathrm{J} \mathrm{Neu}$ rosci 29, 13850-13859.

[131] McManaman JL, et al. (1991) Skeletal muscle proteins rescue motor neurons from cell death in vivo. Adv Neurol 56, 81-88.

[132] Guettier-Sigrist S, et al. (2002) Possible pathogenic role of muscle cell dysfunction in motor neuron death in spinal muscular atrophy. Muscle Nerve 25, 700-708.

[133] Dobrowolny G, et al. (2008) Skeletal muscle is a primary target of SOD1G93A-mediated toxicity. Cell Metab 8, 425-436.

[134] Wong M, Martin LJ (2010) Skeletal muscle-restricted expression of human SOD1 causes motor neuron degeneration in transgenic mice. Hum Mol Genet 19, 2284-2302.

[135] Miller TM, et al. (2006) Gene transfer demonstrates that muscle is not a primary target for non-cell-autonomous toxicity in familial amyotrophic lateral sclerosis. Proc Natl Acad Sci U S A 103, 19546-19551.

[136] Dupuis L, et al. (2003) Up-regulation of mitochondrial uncoupling protein 3 reveals an early muscular metabolic defect in amyotrophic lateral sclerosis. FASEB J 17, 20912093.

[137] Dupuis L, et al. (2009) Muscle mitochondrial uncoupling dismantles neuromuscular junction and triggers distal degeneration of motor neurons. PLoS One 4, e5390.

[138] Dupuis L, et al. (2004) Evidence for defective energy homeostasis in amyotrophic lateral sclerosis: benefit of a high-energy diet in a transgenic mouse model. Proc Natl Acad Sci U S A 101, 11159-11164.

[139] Fergani A, et al. (2007) Increased peripheral lipid clearance in an animal model of amyotrophic lateral sclerosis. $J$ Lipid Res 48, 1571-1580.

[140] Mattson MP, et al. (2007) Energy intake and amyotrophic lateral sclerosis. Neuromolecular Med 9, 17-20.

[141] Vielhaber S, et al. (1999) Visualization of defective mitochondrial function in skeletal muscle fibers of patients with sporadic amyotrophic lateral sclerosis. J Neurol Sci 169, 133-139.

[142] Wiedemann FR, et al. (1998) Impairment of mitochondrial function in skeletal muscle of patients with amyotrophic lateral sclerosis. J Neurol Sci 156, 65-72.

[143] Zhou J, et al. (2010) Hyperactive intracellular calcium signaling associated with localized mitochondrial defects in skeletal muscle of an animal model of amyotrophic lateral sclerosis. J Biol Chem 285, 705-712.

[144] Kawamata H, Manfredi G. Mitochondrial dysfunction and intracellular calcium dysregulation in ALS. Mech Ageing Dev 131, 517-26.

[145] Duffy LM, et al. (2011) Review: the role of mitochondria in the pathogenesis of amyotrophic lateral sclerosis. Neuropathol Appl Neurobiol 37, 336-352.

[146] Wei R, et al. (2012) Protein misfolding, mitochondrial dysfunction and muscle loss are not directly dependent on soluble and aggregation state of mSOD1 protein in skeletal muscle of ALS. Biochem Biophys Res Commun 417, 1275-1279.

[147] Onesto E, et al. (2011) Muscle cells and motoneurons differentially remove mutant SOD1 causing familial amyotrophic lateral sclerosis. J Neurochem 118, 266-280. 
[148] Lokireddy S, et al. (2011) Myostatin promotes the wasting of human myoblast cultures through promoting ubiquitin-proteasome pathway-mediated loss of sarcomeric proteins. Am J Physiol Cell Physiol 301, C1316-C1324.

[149] Purintrapiban J, et al. (2003) Degradation of sarcomeric and cytoskeletal proteins in cultured skeletal muscle cells. Comp Biochem Physiol B Biochem Mol Biol 136, 393-401.

[150] Bonaldo P, Sandri M (2013) Cellular and molecular mechanisms of muscle atrophy. Dis Model Mech 6, 25-39.

[151] Chang Y, et al. (2008) Messenger RNA oxidation occurs early in disease pathogenesis and promotes motor neuron degeneration in ALS. PLoS One 3, e2849.

[152] Williams AH, et al. (2009) MicroRNA-206 delays ALS progression and promotes regeneration of neuromuscular synapses in mice. 326, 1549-1554.

[153] Liu N, et al. (2012) microRNA-206 promotes skeletal muscle regeneration and delays progression of Duchenne muscular dystrophy in mice. J Clin Invest 122, 2054-2065.

[154] Nakasa T, et al. (2010) Acceleration of muscle regeneration by local injection of muscle-specific microRNAs in rat skeletal muscle injury model. J Cell Mol Med 14, 2495-2505.

[155] Williams AH, et al. (2009) MicroRNA-206 delays ALS progression and promotes regeneration of neuromuscular synapses in mice. Science 326, 1549-1554.

[156] Asakura A, et al. (2001) Muscle satellite cells are multipotential stem cells that exhibit myogenic, osteogenic, and adipogenic differentiation. Differentiation 68, 245-253.

[157] Seale P, Rudnicki MA (2000) A new look at the origin, function, and "stem-cell" status of muscle satellite cells. Dev Biol 218, 115-124.

[158] Cooper RN, et al. (1999) In vivo satellite cell activation via Myf5 and MyoD in regenerating mouse skeletal muscle. J Cell Sci 112(Pt 1), 2895-2901.

[159] Yablonka-Reuveni Z, Rivera AJ (1994) Temporal expression of regulatory and structural muscle proteins during myogenesis of satellite cells on isolated adult rat fibers. Dev Biol 164, 588-603.

[160] Smith CK, et al. (1994) Temporal expression of myogenic regulatory genes during activation, proliferation, and differentiation of rat skeletal muscle satellite cells. J Cell Physiol 159, 379-385.

[161] Brack AS, et al. (2007) Increased Wnt signaling during aging alters muscle stem cell fate and increases fibrosis. Science 317, 807-810.

[162] Shefer G, et al. (2006) Satellite-cell pool size does matter: defining the myogenic potency of aging skeletal muscle. Dev Biol 294, 50-66.

[163] Yablonka-Reuveni Z, Anderson JE (2006) Satellite cells from dystrophic (mdx) mice display accelerated differentiation in primary cultures and in isolated myofibers. Dev Dyn 235, 203-212.

[164] Reimann J, et al. (2000) Regenerative capacity and the number of satellite cells in soleus muscles of normal and mdx mice. Neuromuscul Disord 10, 276-282.

[165] Dupuis L, Echaniz-Laguna A (2010) Skeletal muscle in motor neuron diseases: therapeutic target and delivery route for potential treatments. Curr Drug Targets 11, 12501261.

[166] Manzano R, et al. (2012) Quantity and activation of myofiber-associated satellite cells in a mouse model of amyotrophic lateral sclerosis. Stem Cell Rev 8, 279-287.
[167] Kirkinezos IG, et al. (2003) Regular exercise is beneficial to a mouse model of amyotrophic lateral sclerosis. Ann Neurol 53, 804-807.

[168] Drory VE, et al. (2001) The value of muscle exercise in patients with amyotrophic lateral sclerosis. 191.

[169] Mattson MP (2000) Neuroprotective signaling and the aging brain: take away my food and let me run. Brain Res 886, 47-53.

[170] Van Praag H, et al. (1999) Running increases cell proliferation and neurogenesis in the adult mouse dentate gyrus. Nat Neurosci 2, 266-270.

[171] Carro E, et al. (2001) Circulating insulin-like growth factor I mediates the protective effects of physical exercise against brain insults of different etiology and anatomy. $J$ Neurosci 21, 5678-5684.

[172] Knaepen K, et al. (2010) Neuroplasticity - exerciseinduced response of peripheral brain-derived neurotrophic factor: a systematic review of experimental studies in human subjects. Sports Med 40, 765-801.

[173] Winter B, et al. (2007) High impact running improves learning. Neurobiol Learn Mem 87, 597-609.

[174] Stokes KA, et al. (2010) Brief, high intensity exercise alters serum ghrelin and growth hormone concentrations but not IGF-I, IGF-II or IGF-I bioactivity. Growth Horm IGF Res 20, 289-294.

[175] Koziris LP, et al. (1999) Serum levels of total and free IGF-I and IGFBP-3 are increased and maintained in longterm training. J Appl Physiol 86, 1436-1442.

[176] Itoh H, et al. (1998) Effects of endurance physical training on hydroxyl radical generation in rat tissues. Life Sci $\mathbf{6 3}$, 1921-1929.

[177] Dal Bello-Haas V, Florence JM, (2013) Therapeutic exercise for people with amyotrophic lateral sclerosis or motor neuron disease. Cochrane Database Syst Rev 5, CD005229.

[178] Ruscheweyh R, et al. (2011) Physical activity and memory functions: an interventional study. Neurobiol Aging 32, 1304-1319.

[179] Schulz K-H, et al. (2004) Impact of aerobic training on immune-endocrine parameters, neurotrophic factors, quality of life and coordinative function in multiple sclerosis. J Neurol Sci 225, 11-18.

[180] Chiò A, et al. (2005) Severely increased risk of amyotrophic lateral sclerosis among Italian professional football players. Brain 128, 472-476.

[181] Strickland D, et al. (1996) Physical activity, trauma, and ALS: a case-control study. Acta Neurol Scand 94, 45-50.

[182] Longstreth WT, et al. (1991) Hypotheses to explain the association between vigorous physical activity and amyotrophic lateral sclerosis. Med Hypotheses 34, 144-148.

[183] Kurtzke JF (1991) Risk factors in amyotrophic lateral sclerosis. Adv Neurol 56, 245-270.

[184] Li W, et al. (2007) Muscle-derived but not centrally derived transgene GDNF is neuroprotective in G93ASOD1 mouse model of ALS. Exp Neurol 203, 457-471.

[185] Mohajeri MH, et al. (1999) Intramuscular grafts of myoblasts genetically modified to secrete glial cell line-derived neurotrophic factor prevent motoneuron loss and disease progression in a mouse model of familial amyotrophic lateral sclerosis. Hum Gene Ther 10, 1853-1866.

[186] Suzuki M, et al. (2008) Direct muscle delivery of GDNF with human mesenchymal stem cells improves motor neuron survival and function in a rat model of familial ALS. Mol Ther 16, 2002-2010. 
[187] Dobrowolny G, et al. (2005) Muscle expression of a local Igf-1 isoform protects motor neurons in an ALS mouse model. J Cell Biol 168, 193-199.

[188] Kaspar BK, et al. (2003) Retrograde viral delivery of IGF1 prolongs survival in a mouse ALS model. Science 301, 839-842.

[189] Ikemoto A, et al. (2002) Differential expression between synaptic vesicle proteins and presynaptic plasma membrane proteins in the anterior horn of amyotrophic lateral sclerosis. Acta Neuropathol 103, 179-187.

[190] Matsumoto S, et al. (1994) Synaptic pathology of spinal anterior horn cells in amyotrophic lateral sclerosis: an immunohistochemical study. J Neurol Sci 125, 180-185.

[191] Gillingwater TH, Wishart TM (2013) Review: mechanisms underlying synaptic vulnerability and degeneration in neurodegenerative disease. DOI: 10.1111/nan.12014

[192] Cozzolino M, et al. (2012) Amyotrophic lateral sclerosis: new insights into underlying molecular mechanisms and opportunities for therapeutic intervention. Antioxid Redox Signal 17, 1277-1330.

[193] Barber SC, Shaw PJ (2010) Oxidative stress in ALS: key role in motor neuron injury and therapeutic target. Free Radic Biol Med 48, 629-641.

[194] Suzuki H, et al. (2009) ALS-linked P56S-VAPB, an aggregated loss-of-function mutant of VAPB, predisposes motor neurons to ER stress-related death by inducing aggregation of co-expressed wild-type VAPB. J Neurochem $\mathbf{1 0 8}$, 973-985.

[195] Ying Z, et al. (2009) Gp78, an ER associated E3, promotes SOD1 and ataxin-3 degradation. Hum Mol Genet 18, 42684281 .
[196] Ilieva EV, et al. (2007) Oxidative and endoplasmic reticulum stress interplay in sporadic amyotrophic lateral sclerosis. Brain 130, 3111-3123.

[197] Kerman A, et al. (2010) Amyotrophic lateral sclerosis is a non-amyloid disease in which extensive misfolding of SOD1 is unique to the familial form. Acta Neuropathol 119, 335-344.

[198] Prudencio M, et al. (2009) Variation in aggregation propensities among ALS-associated variants of SOD1: correlation to human disease. Hum Mol Genet 18, 3217 3226.

[199] Pascual ML, et al. (2012) Synaptic activity regulated mRNA-silencing foci for the fine tuning of local protein synthesis at the synapse. Commun Integr Biol 5, 388-392.

[200] Otomo A, et al. (2012) Dysregulation of the autophagyendolysosomal system in amyotrophic lateral sclerosis and related motor neuron diseases. Neurol Res Int 2012, 498428

[201] Yamazaki M, et al. (2005) Is motoneuronal cell death in amyotrophic lateral sclerosis apoptosis? Neuropathology 25, 381-387.

[202] Embacher N, et al. (2001) Apoptosis signals in sporadic amyotrophic lateral sclerosis: an immunocytochemical study. Acta Neuropathol 102, 426-434.

[203] Sathasivam S, et al. (2001) Apoptosis in amyotrophic lateral sclerosis: a review of the evidence.

[204] Gould TW, et al. (2006) Complete dissociation of motor neuron death from motor dysfunction by Bax deletion in a mouse model of ALS. $J$ Neurosci 26, 8774-8786.

[205] Barabási A-L (2007) Network medicine-from obesity to the "diseasome". N Engl J Med 357, 404-407. 